\title{
ASSOCIATIONS BETWEEN MULTIDOMAIN LIFESTYLE INTERVENTIONS AND INTRINSIC CAPACITY DOMAINS DURING AGING: A NARRATIVE REVIEW
}

\author{
M. Fourteau ${ }^{1}$, K. Virecoulon Giudici ${ }^{1}$, Y. Rolland ${ }^{1,2}$, B. Vellas ${ }^{1,2}$, P. de Souto Barreto ${ }^{1,2}$
}

\begin{abstract}
Background: Recently, the World Health Organization defined five domains of intrinsic capacity (IC), composed of physical and mental capacities linked to body functions, and that contribute to healthy aging: locomotion, cognition, psychological, vitality and sensorial. In the past decade, studies investigating the effects of concomitant lifestyle interventions (also called multidomain interventions) on one or several IC domains have been developed. The aim of this study is to synthetize the scientific literature about the associations between multidomain lifestyle interventions and IC domains. Methods: We conducted a narrative review of randomized controlled trials examining the effects of multidomain lifestyle interventions on at least one IC domain among older people. Multidomain intervention was defined as the presence of at least two of the following lifestyle interventions: physical activity/ exercise, nutrition, cognitive stimulation, and management of cardiovascular risk factors (eg, smoking, alcohol consumption). Results: Multidomain interventions were associated with improvements on locomotion (as measured by performance-based tests of lower-limb function) and vitality (as measured by handgrip strength); benefits on cognitive function were also found, in particular among populations at increased risk of dementia and when operationalizing strong multidomain interventions (eg, using regular exercise training instead of physical activity advices). No study investigated the effects of multidomain lifestyle interventions on the sensorial domain (hearing and/or vision). The modalities composing the multidomain interventions and intervention length, as well as study population, substantially varied across studies; the most common combination of interventions was physical activity- and nutritional-related interventions. Conclusion: Available evidence is still limited, but literature suggests a positive effect of multidomain lifestyle interventions on IC domains, in particular locomotion. Further studies are still needed on this topic, in particular, studies exploring the effects of multidomain lifestyle interventions on the sensorial domain, as well as on a composite measurement of all IC domains.
\end{abstract}

Key words: Intrinsic capacity, aging, multidomain intervention, locomotion, cognition, psychological, vitality, vision, hearing.

Abbreviations: ADL: Activity of Daily Living; CAIDE: Cardiovascular Risk Factors, Aging, and Incidence of Dementia; FINGER: Finnish Geriatric Intervention Study to Prevent Cognitive Impairment and Disability; FIT: Frailty Intervention Trial; IADL: Instrumental Activities of Daily Living; IC: Intrinsic Capacity; MAPT: Multidomain Alzheimer Preventive Trial; MDI: Multidomain Intervention pre; DIVA: Preventive of Dementia by Intensive Vascular care; RCT: Randomized Controlled Trial; SPPB: Short Physical Performance Battery; WHO: World Health Organization.

\section{Introduction}

Functional decline often occurs during aging. Recently, the World Health Organization (WHO) (1) supported the idea that healthy aging should not be defined as the absence of diseases, but as a process to develop and maintain functional abilities during aging.

1. Gerontopole of Toulouse, Institute of Ageing, Toulouse University Hospital (CHU Toulouse), Toulouse, France; 2. UPS/Inserm UMR1027, University of Toulouse III, Toulouse, France.

Corresponding Author: Marie Fourteau, Gérontopôle de Toulouse, Institut du Vieillissement, 37 Allées Jules Guesde, F-31000 Toulouse, France, Phone: (+33) 561 145 664, Fax: (+33) 561145 640, e-mail: mariefourteau@hotmail.com
In this context, experts from $\mathrm{WHO}$, in collaboration with academic researchers around the world, developed the theoretical framework of intrinsic capacity (IC) (2), ie, the combination of all physical and mental capacities of an individual. These experts proposed to divide IC through five domains that strongly contribute to healthy aging $(1,2)$ : psychological, cognitive, locomotion, vitality and sensory. Therefore, developing strategies that benefit multiple domains of IC would probably lead to the promotion of healthy aging.

Several lifestyle interventions have been found to benefit specific domains of IC. Physical exercise improves locomotion (3) and may improve cognitive function (4, 5 ) as well as psychological outcomes, whereas cognitive training improves cognitive function6; nutritional aspects 
Table 1

Characteristic of the included studies

\begin{tabular}{|c|c|c|c|c|}
\hline Study & Country & Population & Age & Sex (\% women) \\
\hline Kim and al. (18) & $\mathrm{JPN}^{\mathrm{b}}$ & Frail older women. Community-dwelling. & 75 years and older & $100 \%$ \\
\hline Kwon and al. (20) & JPN & Pre-frail women. Community-dwelling. & 70 years and older & $100 \%$ \\
\hline Rydwick and al. (21) & $S W E^{d}$ & Frail elderly people. Community-dwelling. & 75 years and older & $60 \%$ \\
\hline $\begin{array}{l}\text { Cameron and al. }(15,16) \\
\text { Fairhall and al. }(34)\end{array}$ & AUS $^{f}$ & $\begin{array}{l}\text { Frail older people. } \\
\text { Community-dwelling. }\end{array}$ & 70 years and older. & $68 \%$ \\
\hline $\begin{array}{l}\text { Ngaudu and al. (27) } \\
\text { Stephen and al. (28) } \\
\text { Kulmala and al. (35) }\end{array}$ & FIN ${ }^{g}$ & Elderly people with cognitive decline risk & $60-77$ years & $46 \%$ \\
\hline $\begin{array}{l}\text { Moll van Charante and al. (29) } \\
\text { Van Middelaar and al. (30) }\end{array}$ & NLD $^{\mathrm{i}}$ & Elderly participants. Community-dwelling. & $70-78$ years & $54 \%$ \\
\hline Berggren and al. (23) & SWE & Patients with femoral neck fractures & 70 years and older & $74 \%$ \\
\hline Lee and al. (37) & $\mathrm{KOR}^{j}$ & Community-dwelling older adults & 60 years and older & $78 \%$ \\
\hline Barnes and al. (36) & USA & Elderly people with memory complaint & 65 years and older & $62 \%$ \\
\hline
\end{tabular}

a. Singapore; b. Japan; c. Taïwan; d. Sweden; e. Austria; f. Australia; g. Finland; h. France; i. Holland; j. Korea;

are also associated with different IC domains $(7,8)$. From the observation that different lifestyle interventions may lead to improvements in different clinical outcomes, the benefits of the combination of different lifestyle interventions, the so-called "multidomain intervention", have been recently explored. Multidomain lifestyle interventions would potentially have synergistic and positive effects on IC domains. However, as far as we know, no study gathered the available scientific evidence of the effects of multidomain lifestyle interventions on the five domains of IC. Moreover, the best combination of lifestyle interventions to improve older adults' function remains to be elucidated.

This narrative review aimed to synthetize the scientific findings regarding the effects of randomized controlled trials (RCT) of multidomain lifestyle interventions on the domains of IC among older adults.

\section{Methods}

\section{Eligibility criteria}

Articles were eligible for this review if they were written in English or French; reported the results of a RCT regarding the effects of multidomain lifestyle interventions on one or several IC domains; included older adults (participants' minimum age or the mean age of the study population $\geq 60$ years). Multidomain lifestyle intervention was defined as the concomitant presence of at least two of the following lifestyle interventions: physical activity/exercise, nutrition, cognitive stimulation, and management of cardiovascular risk factors (eg, smoking, alcohol consumption).

The domains of IC were defined as follows:

1. Locomotion. Measured using the Short Physical Performance Battery9 (SPPB) or gait speed.

2. Cognition. Measured using a validated neuropsychological test or a battery of tests for older adults.

3. Psychological. Measured using a validated scale of depressive symptoms for the elderly (such as the Geriatric Depression Scale - GDS).

4. Vitality. A consensual way of measuring the concept of vitality is not yet established10. We opted to operationalize this domain by using the handgrip strength10, which is a vital sign during aging11 and is associated to nutritional status12.

5. Sensory. Measured using validated tests for vision (eg, near/distance visual acuity) and hearing (eg, audiometry, Whisper test) capacities in older people.

The exclusion criteria comprised studies using a single lifestyle intervention or comparing different types of lifestyle interventions without assessing the effects of the combination of at least two interventions; mean age of the 


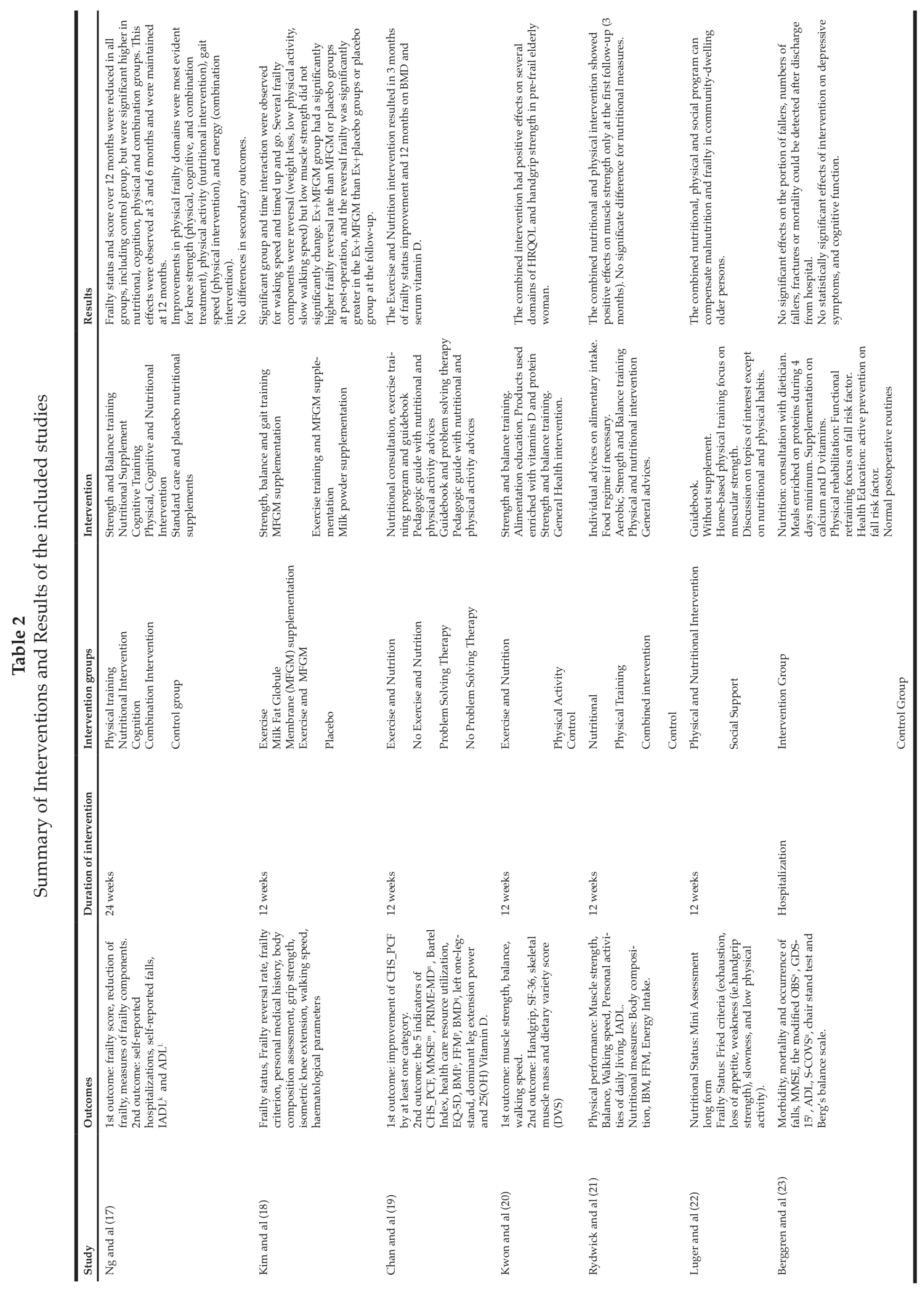




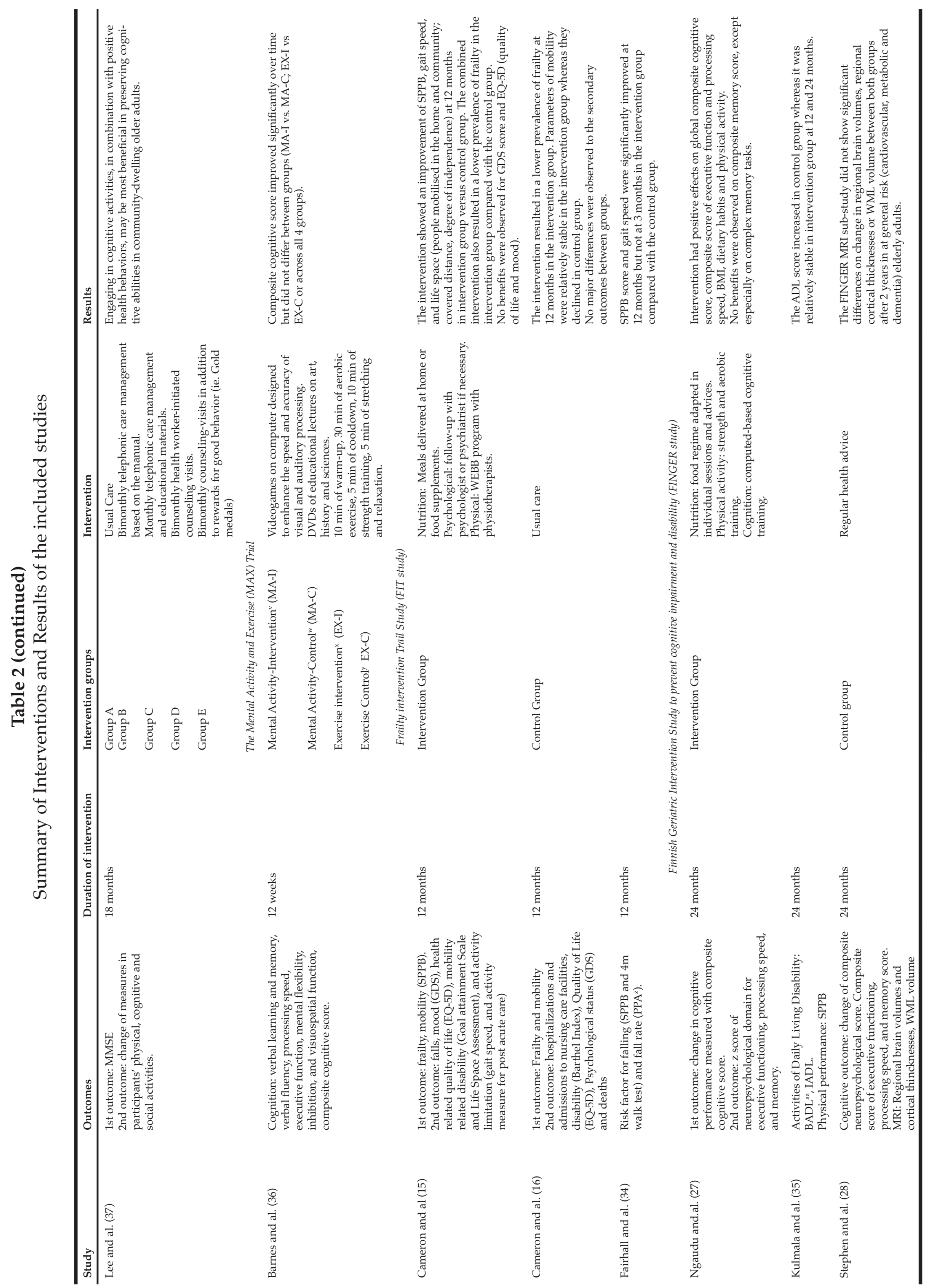




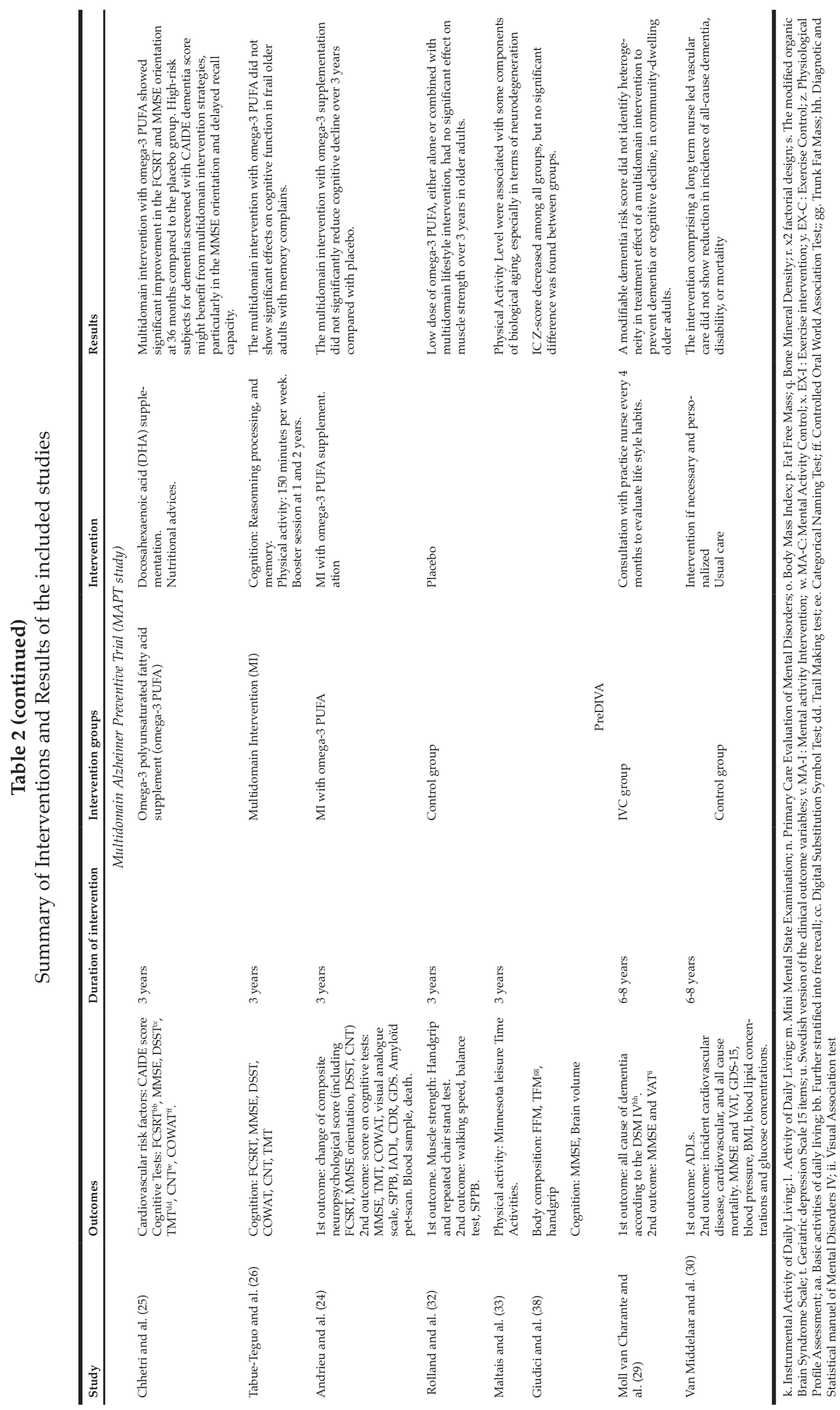


study population < 60 years; and samples of participants specifically presenting $\mathrm{MCI}$ or dementia.

\section{Search strategy}

Potentially eligible studies were searched on PubMed database from November 2019 to July 2020, and in the reference lists of previous literature reviews and other publications $(13,14)$, as well as authors' personal files. For the search, key-words related to the population (eg, elderly), the intervention (eg, multidomain), and the study design (eg, random) were used. All the search terms are summarized in Supplementary Table 1.

\section{Data extraction}

One author extracted the information of selected publications on: study population characteristics, intervention, IC domains investigated, and findings of the effects of multidomain lifestyle intervention on the IC domain. This review included 23 articles. Characteristics of key articles/studies and interventions are, respectively, presented in Table 1 and Table 2 (15-38).

\section{Results}

The articles/studies were performed in Japan $(18,20)$, Australia (15, 16), Korea (37), Singapore (17), Taiwan (19), Austria (22), Finland (21, 27, 28, 35), France (24-26, $32,33,38)$, the Netherlands $(29,30)$, the United States (36) and Sweden $(21,23)$. The sample size varied from 80 to 1680 participants across studies, with a mean age higher than 65 years old for all studies. Intervention length varied from 2 months to 6 years. The most used multidomain lifestyle intervention was a combination of physical activity, nutritional and cognitive interventions $(17,24-28,35,38)$; a combination of physical activity and nutritional intervention (18-21) or individuallytailored multifactorial interventions (including exercise, nutritional and psychological interventions depending on individual's needs) $(15,16,34)$. A combination of physical activity and cognitive intervention (36) or physical activity, nutritional intervention and health education (22, 23); as well as assessments of cardiovascular risk factor and adapted advice were also observed $(29,30)$. Thus, the advices and encouragement in physical, cognitive and social activities, in combination with rewards for good health behaviours (37) were included.

About the IC domains investigated, locomotion $(15-18,20,21,24,27,34,35)$ and cognition (23-30, $36,37)$ capacities were the most frequent IC domains investigated, followed by the vitality domain (as measured by handgrip strength) $(16,18-20,22,32)$, and then the psychological domain $(15,16,19,23,27,33)$. One study has investigated the effects of a multidomain lifestyle intervention on a composite score of IC (38.) This analysis, using data of the MAPT study, has included the following four IC domains: locomotion, cognition, psychological and vitality.

No study has assessed the effects of multidomain lifestyle interventions on a valid measurement of vision or hearing capacities (sensorial domain).

Effects of multidomain lifestyle intervention on IC domains in elderly persons

\section{IC Z-score}

Recently, one study from MAPT38 has investigated the effects of a multidomain lifestyle intervention (composed by physical activity advices, nutritional counselling, and cognitive training), with or without omega-3 supplementation, on an IC Z-score including four domains (ie. locomotion, psychology, cognition and vitality). After three years, the IC Z-score decreased among all groups, but no significant difference was found between groups.

This article was the first to study the effects of a multidomain lifestyle intervention on a composite score of intrinsic capacity. Further studies are needed in order to be able to conclude on this topic.

\section{Locomotion}

Some publications (15-18, 20, 21, 24, 27, 34, 35) evaluated the effects of multidomain lifestyle interventions on locomotion. Regarding gait speed, results of at least three articles $(15,18,34)$ showed an improvement of gait speed in the multidomain intervention group. This significant increase was observed in particular among participants with slow gait speed and participants with higher levels of frailty. Besides, the combined physical, nutritional and psychological intervention (15) showed also an improvement of gait speed in frailty older adults. However, other studies $(17,20,21,24)$ did not find any effects of multidomain intervention on walking speed.

At least five articles $(15,16,24,34,35)$ used the SPPB score in order to measure the mobility of participants. The multidomain approach composed of individuallytailored interventions (including exercise, nutritional, and psychosocial support depending on individual's needs) described in three publications $(15,16,34)$ had positive effects on the SPPB score after 12 months, but not at 3 months of follow-up. Additionally, the combination of physical activity, nutritional counselling, cognitive training, social activities and management of metabolic and vascular risk in the Finnish Geriatric Intervention Study to Prevent Cognitive Impairment and Disability (FINGER) (35) showed a protective effect of physical decline for chair rise test, but no effects were observed for SPPB score (27). However, another study with an intervention composed by physical activity, nutritional counselling and cognitive training, with or without omega-3 polyunsaturated fatty acid supplementation, had 
no effects on the SPPB score (24).

Overall, despite still limited findings, part of the currently scientific evidence about SPPB supports a positive effect of multidomain lifestyle intervention. In contrast, concerning gait speed, most of the included studies found no effects of multidomain lifestyle intervention.

\section{Cognition}

Several articles $(23-30,36,37)$ assessed cognitive capacity after a multidomain intervention. The assessment of cognitive function varied across studies. Executive function was an outcome in two studies $(26,27)$, and was improved in one of them (27). In the FINGER Study (27), both intervention and control groups (usual health advice) had an increase on cognitive function (executive function and processing speed), but this improvement was significantly higher in the intervention group after 24 months. Despite using magnetic resonance imaging (MRI) (28), authors were unable to explain the effects of this multidomain intervention on global cognitive function by some structural brain mechanisms. One study (37) proposing bimonthly counseling-visits with rewards for good behavior showed superior cognitive function (ie. improvement of MMSE score) compared to the control group (ie. standards and usual care), identifying participation at cognitive activities as a protector factor against cognitive decline. The Mental Activity and eXercise (MAX) Trial (36) enrolled inactive older adults with cognitive complaints in home-based mental activity and class-based physical activity for 12 weeks, and found an improvement of global cognitive scores over time but with no differences between intervention and active control groups, what may suggest that, for this population, the amount of activity would be more important than its type.

Moreover, analysis of the effects of interventions in the Multidomain Alzheimer Preventive Trial (MAPT study) (25) restricted to the subgroup of older adults with higher risk of dementia due to cardiovascular risk factors (CAIDE dementia risk score) showed an improvement of the cognitive function in multidomain groups; similar results were found in the subgroup with high $\beta$-amyloid load in the brain (24). However, it is important to highlight that in the full sample of the MAPT study (24) as well as in the Preventive of Dementia by Intensive Vascular care (preDIVA) $(29,30)$ no effects were observed on cognitive function, including among those with an increased dementia risk score. Besides, the addition of rewards for good behaviours at the combined nutritional and cognitive activities (23) did not show beneficial effect on cognitive function.

Therefore, the effects of multidomain lifestyle intervention on cognitive function are not a consensus. On the one hand, two studies presented positive effects for this type of intervention. These results corroborate with those of another study comparing the effects of physical activity, cognitive training and the combination of both with a control group (39). On the other hand, two cohorts did not find any results on cognitive function (24, $25,29,30)$. Thus, the effectiveness of multidomain lifestyle interventions on cognitive function still demands further exploring.

\section{Psychological}

Only few studies $(15,16,19,23,27,33)$ tested the effects of multidomain interventions on psychological function among elderly subjects. The results of these studies (15, $16,19,23,27,33)$ revealed no effects of multidomain interventions on depressive symptoms (measured by the GDS or the Center for Epidemiologic Studies Depression Scale - CES-D) in elderly people with frailty, memory complains or at risk of cognitive decline, after follow-ups varying from 3 months to 3 years.

So far, the available scientific evidence did not support any effects of multidomain intervention on the psychological domain of IC among older adults.

\section{Vitality}

Some studies of multidomain RCT $(16,18-20$, 22,32 ) evaluated the effects of the intervention on handgrip strength. At least three studies showed significant positive effects of interventions - including individually-tailored interventions (physical activity, nutritional intervention, psycho-social support according to participants' needs) (16); exercise and cognitive intervention (19); and exercise associated with nutritional intervention (22) - on handgrip strength. Effects were significant in follow-ups of 12 months or over.

We found two studies that showed no improvement from the multidomain intervention (including exercise training and nutritional supplementation of milk fat globule membrane (18); or physical activity, nutritional counselling, and cognitive training (32)) on handgrip strength. However, one study (20) comprising nutritional and physical intervention showed a decline in handgrip during post-intervention follow-up, after maintenance during the combined intervention.

In summary, effects of multidomain lifestyle intervention on handgrip strength in older adults are still mixed. Twos studies found positive effects, other two studies found no effects on vitality domain and one study did not find significant benefits on handgrip during intervention but showed a significant decline postintervention. Thus, it is not possible to conclude on the effectiveness of multidomain lifestyle interventions on handgrip measure. 


\section{Final considerations}

This is the first review focused on current evidence of the effects of multidomain lifestyle interventions on IC among elderly people. One of the findings revealed herein is the complete absence of studies considering the sensory domain (hearing and vision) as an outcome. Although positive effects of multidomain interventions on locomotion, cognition and vitality (handgrip strength) were observed, findings were globally mixed. Another important finding of this review is that, as far as we know, only one study investigated the effects of the multidomain intervention on a global IC score, operationalized by considering four of the five domains of IC, except the sensory domain (38). The limited evidence about this topic does not allow us to conclude whether or not multidomain interventions would globally affect intrinsic capacity. Further studies operationalizing IC are therefore necessary.

The absence of multidomain RCT examining intervention effects on the sensorial IC domain is understandable given the nature of this domain: improving vision and hearing would not be expected with nutritional counselling or stimulating physical activity, for example. However, it is possible that multidomain interventions could contribute to preventing sensorial impairments, in particular for vision, through the prevention and control of cardiovascular and metabolic conditions, such as hypertension and diabetes. The well-known associations of physical activity/exercise and the prevention/management of cardiovascular and metabolic diseases are well-established (40-42). In addition, studies including antioxidant supplementations $(43,44)$, or multidomain lifestyle intervention (including Mediterranean diet, physical activity, avoided smoking and sedentary behaviours) (45) suggested positive effects on the progress and prevalence of aging related macular degeneration. It can also be noted that in MAPT Study, preventive consultations (which were part of the multidomain intervention) included evaluation of vision and hearing deficits, with recommendations for management where necessary (46).

Although still limited, available evidence supports an effect of multidomain interventions on locomotion when exercise training is present. SPPB was improved in multidomain RCTs that operationalized exercise sessions (not only advices on physical activity), even though other trials with exercise sessions found no effects on locomotion outcomes. It is possible that multidomain interventions have an effect on global locomotion (SPPB) only when a comprehensive exercise training, with strength but also balance exercises, is comprised in the intervention; indeed, a multidomain trial (34), including exercise training with strength and balance exercises, had positive effects on SPPB, in particular among frail participants and those who were more compliant with the intervention.
Multidomain lifestyle interventions improved cognition among older adults when operationalizing strong interventions (27) or for subpopulation of individuals at increased risk of cognitive decline (25, 47). Indeed, the FINGER trial (27) had supervised strength and aerobic training, compared to other RCTs that restricted the physical part of the intervention to counselling (24). Moreover, FINGER (27) and MAPT (24), this latter showing positive effects of the multidomain intervention on cognition among people at high risk for dementia (ie, high both CAIDE dementia score and amyloid load in the brain), had strong cognitive training and were among the largest (well-powered) and longest multidomain RCTs. Therefore, it is possible that lifestyle multidomain interventions should have strong components for both cognitive and exercise training to increase cognitive function. Multidomain interventions may still benefit cognition with a less strong physical component in subpopulations at increased risk for clinically meaningful cognitive decline.

For the psychological IC domain, we did not find improvements of multidomain interventions on depressive symptoms $(15,19,23,33)$. It is possible that in order to improve the psychological domain of IC, an intervention with more important psychological content (eg, group-based activities focusing on social support) would be needed. It is also plausible to think, as it seems to occur for the cognitive IC domain, that multidomain lifestyle interventions would be more effective in a subpopulation at increased risk for depression.

The handgrip strength, composing the vitality domain, was improved in three studies $(16,19,22)$, but not in two others trials $(18,32)$; therefore, literature on this topic is mixed. One major point regards the non-consensual definition of vitality: although we opted to operationalise this IC domain using handgrip strength, as in previous publications (10), another operational definition using nutritional status is attracting further attention, including the World Health Organization (48). Although handgrip is associated with nutritional status (12), they are distinct measurements. It is thus possible that nutritional status would respond differently to a multidomain lifestyle intervention; this is still truer since a nutritionrelated component is found in nearly all multidomain interventions operationalized to date.

Taken together, findings suggest that, in the long term, multidomain interventions can bring beneficial effects to health. The magnitude of these benefits will vary according to the modalities of intervention composing the multidomain approach. Globally, long-term (one year or over) and strong (eg, composed by exercise training instead of physical activity counselling) interventions lead to benefits on specific IC domains. It should be noted, however, that this review found an important variability across studies regarding the modalities of intervention composing the multidomain intervention. This variability makes it difficult to compare their findings and to generalize their results. Moreover, the 
small quantity of studies with multidomain interventions in elderly people do not allow us to precisely define the more effective protocol to each IC domain. Such methodological differences may probably have contributed to the mixed findings gathered in the present work.

\section{Conclusion}

IC tend to decline during aging, reducing individuals' resilience and increasing their vulnerability to adverse health outcomes. Multidomain interventions are an interesting approach to optimize the effects of combined lifestyle interventions on the different IC domains. This review gathered heterogeneous findings on the effects of multidomain interventions on the different IC domains. Although still limited, the evidence suggest multidomain interventions may benefit locomotion and, to a lesser extent, cognition and vitality (handgrip strength). Developing strategies for preserving IC is crucial and of high clinical interest in the scenery of integrated care for older adults. Therefore, further investigating the links of multidomain interventions with each IC domain, but also with a global measurement of IC (combining all domains) would importantly contribute to the topic.

Acknowledgments: The present work was performed in the context of the Inspire Program, a research platform supported by grants from the Region Occitanie/Pyrénées-Méditerranée (Reference number: 1901175) and the European Regional Development Fund (ERDF) (Project number: MP0022856).

Conflict of interest: The author(s) declare(s) that there is no conflict of interest regarding the publication of this article.

Ethical standard: All procedures followed were in accordance with the ethical standards.

\section{References}

1. World Health Organization. World report on ageing and health. WHO. Published 2015. Accessed November 25, 2019. http:/ / www.who.int/ageing/ events/world-report-2015-launch/en/

2. Cesari M, Araujo de Carvalho I, Amuthavalli Thiyagarajan J, et al. Evidence for the Domains Supporting the Construct of Intrinsic Capacity. J Gerontol Ser A. 2018;73(12):1653-1660. doi:10.1093/gerona/gly011

3. Pahor M, Guralnik JM, Ambrosius WT, et al. Effect of Structured Physical Activity on Prevention of Major Mobility Disability in Older Adults: The LIFE Study Randomized Clinical Trial. JAMA. 2014;311(23):2387. doi:10.1001/ jama.2014.5616

4. Young J, Angevaren M, Rusted J, Tabet N. Aerobic exercise to improve cognitive function in older people without known cognitive impairment. Cochrane Dementia and Cognitive Improvement Group, ed. Cochrane Database Syst Rev. Published online April 22, 2015. doi:10.1002/14651858. CD005381.pub4

5. Kelly ME, Loughrey D, Lawlor BA, Robertson IH, Walsh C, Brennan S. The impact of exercise on the cognitive functioning of healthy older adults: A systematic review and meta-analysis. Ageing Res Rev. 2014;16:12-31. doi:10.1016/j.arr.2014.05.002

6. Ball K, Berch DB, Helmers KF, et al. Effects of Cognitive Training Interventions With Older Adults: A Randomized Controlled Trial. JAMA 2002;288(18):2271. doi:10.1001/jama.288.18.2271

7. Struijk EA, Guallar-Castillón P, Rodríguez-Artalejo F, López-García E. Mediterranean Dietary Patterns and Impaired Physical Function in Older Adults. J Gerontol A Biol Sci Med Sci. Published online October 19, 2016:glw208. doi:10.1093/gerona/glw208

8. Loughrey DG, Lavecchia S, Brennan S, Lawlor BA, Kelly ME. The Impact of the Mediterranean Diet on the Cognitive Functioning of Healthy Older Adults: A Systematic Review and Meta-Analysis. :16.

9. Guralnik JM, Simonsick EM, Ferrucci L, et al. A Short Physical Performance
Battery Assessing Lower Extremity Function: Association With Self-Reported Disability and Prediction of Mortality and Nursing Home Admission. J Gerontol. 1994;49(2):M85-M94. doi:10.1093/geronj/49.2.M85

10. Giudici KV, de Souto Barreto P, Soriano G, Rolland Y, Vellas B. Defining Vitality: Associations of Three Operational Definitions of Vitality with Disability in Instrumental Activities of Daily Living and Frailty among Elderly Over a 3-Year Follow-Up (MAPT Study). J Nutr Health Aging. 2019;23(4):386-392. doi:10.1007/s12603-019-1175-0

11. Leong DP, Teo KK, Rangarajan S, et al. Prognostic value of grip strength: findings from the Prospective Urban Rural Epidemiology (PURE) study. The Lancet. 2015;386(9990):266-273. doi:10.1016/S0140-6736(14)62000-6

12. Flood A, Chung A, Parker H, Kearns V, O'Sullivan TA. The use of hand grip strength as a predictor of nutrition status in hospital patients. Clin Nutr Edinb Scotl. 2014;33(1):106-114. doi:10.1016/j.clnu.2013.03.003

13. Dedeyne L, Deschodt M, Verschueren S, Tournoy J, Gielen E. Effects of multidomain interventions in (pre)frail elderly on frailty, functional, and cognitive status: a systematic review. Clin Interv Aging. 2017; Volume 12:873-896. doi:10.2147/CIA.S130794

14. Schneider N, Yvon C. A review of multidomain interventions to support healthy cognitive ageing. J Nutr Health Aging. 2013;17(3):252-257 doi:10.1007/s12603-012-0402-8

15. Cameron ID, Fairhall N, Gill L, et al. Developing Interventions for Frailty. Adv Geriatr. 2015;2015:1-7. doi:10.1155/2015/845356

16. Cameron ID, Fairhall N, Langron C, et al. A multifactorial interdisciplinary intervention reduces frailty in older people: randomized trial. BMC Med. 2013;11(1):65. doi:10.1186/1741-7015-11-65

17. Ng TP, Feng L, Nyunt MSZ, et al. Nutritional, Physical, Cognitive, and Combination Interventions and Frailty Reversal Among Older Adults: A Randomized Controlled Trial. Am J Med. 2015;128(11):1225-1236.e1. doi:10.1016/j.amjmed.2015.06.017

18. Kim H, Suzuki T, Kim M, et al. Effects of Exercise and Milk Fat Globule Membrane (MFGM) Supplementation on Body Composition, Physical Function, and Hematological Parameters in Community-Dwelling Frail Japanese Women: A Randomized Double Blind, Placebo-Controlled, FollowUp Trial. Buchowski M, ed. PLOS ONE. 2015;10(2):e0116256. doi:10.1371/ journal.pone.0116256

19. Chan D-CD, Tsou H-H, Yang R-S, et al. A pilot randomized controlled trial to improve geriatric frailty. BMC Geriatr. 2012;12(1):58. doi:10.1186/1471-2318$12-58$

20. Kwon J, Yoshida Y, Yoshida H, Kim H, Suzuki T, Lee Y. Effects of a Combined Physical Training and Nutrition Intervention on Physical Performance and Health-Related Quality of Life in Prefrail Older Women Living in the Community: A Randomized Controlled Trial. J Am Med Dir Assoc. 2015;16(3):263.e1-263.e8. doi:10.1016/j.jamda.2014.12.005

21. Rydwik E, Lammes E, Frändin K, Akner G. Effects of a physical and nutritional intervention program for frail elderly people over age 75. A randomized controlled pilot treatment trial. Aging Clin Exp Res. 2008;20(2):159-170. doi:10.1007/BF03324763

22. Luger E, Dorner TE, Haider S, Kapan A, Lackinger C, Schindler K. Effects of a Home-Based and Volunteer-Administered Physical Training, Nutritional, and Social Support Program on Malnutrition and Frailty in Older Persons: A Randomized Controlled Trial. J Am Med Dir Assoc. 2016;17(7):671.e9-671.e16. doi:10.1016/j.jamda.2016.04.018

23. Berggren M, Stenvall M, Olofsson B, Gustafson Y. Evaluation of a fallprevention program in older people after femoral neck fracture: a one-year follow-up. Osteoporos Int. 2008;19(6):801-809. doi:10.1007/ s00198-007-0507-9

24. Andrieu S, Guyonnet S, Coley N, et al. Effect of long-term omega 3 polyunsaturated fatty acid supplementation with or without multidomain intervention on cognitive function in elderly adults with memory complaints (MAPT): a randomised, placebo-controlled trial. Lancet Neurol. 2017;16(5):377-389. doi:10.1016/S1474-4422(17)30040-6

25. Chhetri JK, de Souto Barreto P, Cantet C, et al. Effects of a 3-Year MultiDomain Intervention with or without Omega-3 Supplementation on Cognitive Functions in Older Subjects with Increased CAIDE Dementia Scores. J Alzheimers Dis. 2018;64(1):71-78. doi:10.3233/JAD-180209

26. Tabue-Teguo M, Barreto de Souza P, Cantet C, et al. Effect of Multidomain Intervention, Omega-3 Polyunsaturated Fatty Acids Supplementation or their Combinaison on Cognitive Function in Non-Demented Older Adults According to Frail Status: Results from the MAPT Study. J Nutr Health Aging. 2018;22(8):923-927. doi:10.1007/s12603-018-1024-6

27. Ngandu T, Lehtisalo J, Solomon A, et al. A 2 year multidomain intervention of diet, exercise, cognitive training, and vascular risk monitoring versus control to prevent cognitive decline in at-risk elderly people (FINGER): a randomised controlled trial. The Lancet. 2015;385(9984):2255-2263. doi:10.1016/S0140-6736(15)60461-5

28. for the FINGER study group, Stephen R, Liu Y, et al. Brain volumes and cortical thickness on MRI in the Finnish Geriatric Intervention Study to Prevent Cognitive Impairment and Disability (FINGER). Alzheimers Res Ther. 2019;11(1):53. doi:10.1186/s13195-019-0506-z

29. van Charante EPM, Richard E, Eurelings LS, et al. Effectiveness of a 6-year multidomain vascular care intervention to prevent dementia (preDIVA): a cluster-randomised controlled trial. The Lancet. 2016;388(10046):797-805. 
doi:10.1016/S0140-6736(16)30950-3

30. van Middelaar T, Hoevenaar-Blom MP, van Gool WA, et al. Modifiable dementia risk score to study heterogeneity in treatment effect of a dementia prevention trial: a post hoc analysis in the preDIVA trial using the LIBRA index. Alzheimers Res Ther. 2018;10(1):62. doi:10.1186/s13195-018-0389-4

31. the SHARP-P Study Group, Legault C, Jennings JM, et al. Designing clinical trials for assessing the effects of cognitive training and physical activity interventions on cognitive outcomes: The Seniors Health and Activity Research Program Pilot (SHARP-P) Study, a randomized controlled trial. BMC Geriatr. 2011;11(1):27. doi:10.1186/1471-2318-11-27

32. Rolland Y, Barreto P de S, Maltais M, et al. Effect of Long-Term Omega 3 Polyunsaturated Fatty Acid Supplementation with or without Multidomain Lifestyle Intervention on Muscle Strength in Older Adults: Secondary Analysis of the Multidomain Alzheimer Preventive Trial (MAPT). Nutrients. 2019;11(8):1931. doi:10.3390/nu11081931

33. Maltais M, de Souto Barreto P, Pothier K, et al. Lifestyle multidomain intervention, omega-3 supplementation, or both for reducing the risk of developing clinically relevant depressive symptoms in older adults with memory complaints? Secondary analysis from the MAPT trial. Exp Gerontol. 2019;120:28-34. doi:10.1016/j.exger.2019.02.010

34. Fairhall N, Sherrington C, Lord SR, et al. Effect of a multifactorial, interdisciplinary intervention on risk factors for falls and fall rate in frail older people: a randomised controlled trial. Age Ageing. 2014;43(5):616-622. doi:10.1093/ageing/aft204

35. Kulmala J, Ngandu T, Havulinna S, et al. The Effect of Multidomain Lifestyle Intervention on Daily Functioning in Older People: LIFESTYLE INTERVENTION AND DAILY FUNCTIONING. J Am Geriatr Soc. 2019;67(6):1138-1144. doi:10.1111/jgs.15837

36. Barnes DE, Santos-Modesitt W, Poelke G, et al. The Mental Activity and eXercise (MAX) Trial: A Randomized Controlled Trial to Enhance Cognitive Function in Older Adults. JAMA Intern Med. 2013;173(9):797. doi:10.1001/ jamainternmed.2013.189

37. Lee KS, Lee Y, Back JH, et al. Effects of a Multidomain Lifestyle Modification on Cognitive Function in Older Adults: An Eighteen-Month CommunityBased Cluster Randomized Controlled Trial. Psychother Psychosom. 2014;83(5):270-278. doi:10.1159/000360820

38. Giudici KV, de Souto Barreto P, Beard J, et al. Effect of long-term omega3 supplementation and a lifestyle multidomain intervention on intrinsic capacity among community-dwelling older adults: Secondary analysis of a randomized, placebo-controlled trial (MAPT study). Maturitas. 2020;141:39-
45. doi:10.1016/j.maturitas.2020.06.012

39. Shatil E. Does combined cognitive training and physical activity training enhance cognitive abilities more than either alone? A four-condition randomized controlled trial among healthy older adults. Front Aging Neurosci. 2013;5. doi:10.3389/fnagi.2013.00008

40. de Souto Barreto P, Cesari M, Andrieu S, Vellas B, Rolland Y. Physical Activity and Incident Chronic Diseases: A Longitudinal Observational Study in 16 European Countries. Am J Prev Med. 2017;52(3):373-378. doi:10.1016/j. amepre.2016.08.028

41. Wisløff U, Nilsen TIL, Drøyvold WB, Mørkved S, Slørdahl SA, Vatten LJ. A single weekly bout of exercise may reduce cardiovascular mortality: how little pain for cardiac gain? 'The HUNT study, Norway.' Eur I Cardiovasc Prev Rehabil. 2006;13(5):798-804. doi:10.1097/01.hjr.0000216548.84560.ac

42. Moholdt T, Wisløff U, Nilsen TIL, Slørdahl SA. Physical activity and mortality in men and women with coronary heart disease: prospective population-based cohort study in Norway (the HUNT study). Eur J Cardiovasc Prev Rehabil. 2008;15(6):639-645. doi:10.1097/ HJR.0b013e3283101671

43. Hallfrisch J, Mu B. Are Antioxidants or Supplements Protective for AgeRelated Macular Degeneration? :6.

44. Evans JR, Lawrenson JG. Antioxidant vitamin and mineral supplements for slowing the progression of age-related macular degeneration. Cochrane Eyes and Vision Group, ed. Cochrane Database Syst Rev. Published online July 30 2017. doi:10.1002/14651858.CD000254.pub4

45. Carneiro Â, Andrade JP. Nutritional and Lifestyle Interventions for Age-Related Macular Degeneration: A Review. Oxid Med Cell Longev. 2017;2017:1-13. doi:10.1155/2017/6469138

46. Vellas B, Carrie I, Gillette-Guyonnet S, et al. MAPT study : a multidomain approach for preventing Alzheimer's disease : design and baseline data. 2014;1(1):12.

47. Delrieu J, Payoux P, Carrié I, et al. Multidomain intervention and/or omega-3 in nondemented elderly subjects according to amyloid status Alzheimers Dement J Alzheimers Assoc. 2019;15(11):1392-1401. doi:10.1016/j. jalz.2019.07.008

48. WHO | WHO Guidelines on Integrated Care for Older People (ICOPE). WHO. Accessed January 25, 2019. http://www.who.int/ageing/ publications / guidelines-icope/en/ 